\title{
Microwave Assisted Heterocyclization: A Rapid and Efficient Synthesis and Antibacterial Activity of Novel Thiazolidinones
}

\author{
B. D. NAIK and K. R. DESAI* \\ Department of Chemistry, South Gujarat University, \\ Surat - 395 007. India.
}

Received 16 September 2004; Accepted 10 October 2004

\begin{abstract}
As a target to synthesize various Thiazolidinone derivatives, 2-Amino-4(coumarin-3-yl)-thiazole has been prepared by the reactions of 3-Bromo acetyl coumarin with thiourea. 3-Bromo acetyl coumarin was prepared from 3-Acetyl coumarin. The resulting compound 2-amino-4-(coumarin-3-yl)- thiazole was treated with different Aldehydes to give the intermediate Schiff base, which on further reaction with Thioglycolic acid and Thiolactic acid to give titled compound Thiazolidinone. The structures of the compounds have been confirmed by elemental analysis and spectral analysis. The antibacterial activity of the compounds has also been screened against Staphylococcus aureus and Escherichia coli.
\end{abstract}

Key words Schiff Base, Thiazolidinone, Antibacterial activity.

\section{Introduction}

A survey of literature reveals that Thiazolo Coumarins posses a broad spectrum of biological importance. Thiazolo Coumarin derivatives are well known biologically active compounds. Schiff bases from 2-Amino-4- (coumarin-3-yl)-thiazole and various Aldehydes are reported to have significant antibacterial activity. These derivatives have been reported as bacteriostatic ${ }^{1}$ and fungisidic ${ }^{2}$. Schiff Base gives good antimicrobial activity and pharmacological applications ${ }^{3}$ and it can be prepared by the acid catalyzed reaction of amines \& Ketones or Aldehydes. It gives a good fungicidal activity ${ }^{4}$. 4Thiazolidinones gives good pharmacological properties ${ }^{5}$. 4-Thiazolidinones are known to exhibit antitubercular ${ }^{6}$, antibacterial ${ }^{7}$, anticonvulsant ${ }^{8}$, antifungal ${ }^{9}$, and antithyroid activities.

The application of microwave irradiation (MWI) to organic synthesis has been the focus of considerable attention in recent years and is becoming an increasingly popular technology ${ }^{10}$. The salient features of the microwave approach are rapid reaction rates, cleaner reaction conditions and enhancements in chemical yields ${ }^{11-12}$.

Under the framework of "Green Chemistry" we have developed an environmentally benign synthesis of Thiazolidinones. Further attractions of this method are that it allows reactions in open vessels (thus avoiding the risk of high pressure development) and synthesis on preparative scale ${ }^{13}$. Herein we wish to report a facile microwave synthesis of Thiazolidinone from 2-Amino-4-(coumarin-3yl)-thiazole and various aldehydes using DMF as a reaction mediator. 
Different Aldehydes were condensed with 2-Amino-4-(coumarin- 3-yl)-thiazole to yield Schiff base. The Schiff bases were further reacted with Thioglycolic acid and Thiolactic acid to yield Thiazolidinone derivatives $[A] \&[B]$.

\section{Experimental}

All the melting points were determined on a PMP-DM scientific melting point apparatus and are uncorrected. IR spectra were recorded with $\mathrm{KBr}$ on Shimatzu FT-IR 8300 spectrophotometer. Reactions were carried out in a "QPro-M Modified Microwave System" made in CANADA.

Preparation of 2 -(3 -hydroxy-4 -methoxy phenyl)-3 -[4-(coumarin-3-yl)-thiazole-2-yl]-4 thiazolidinone $[\mathrm{A}]$ :

The (Schiff base), 2-N-(3 -methoxy-4 -hydroxy benzylidene) imino-4-(coumarin-3-yl)-thiazole (0.01 mole, $3.78 \mathrm{~g})$ in DMF was taken in R.B.F. To it thioglycolic acid $(0.01$ mole, $0.92 \mathrm{~g})$ was added slowly. Then it was placed inside a microwave oven for about 3.0 minutes. It was then diluted with ice-cold water. The solid product formed was filtered, dried and recrystallised from Ethanol. Other substituted Thiazolidinones were prepared in a similar manner. Data for various substituted Thiazolidinones are given in Table-1.

Preparation of 2 -(3 -hydroxy-4 -methoxy phenyl)-5 -methyl-3 -[4-(coumarin-3-yl)-thiazole-2-yl]4 -thiazolidinone [B]:

The (Schiff base), 2-N-(3 -methoxy-4 -hydroxy benzylidene) imino-4-(coumarin-3-yl)-thiazole (0.01 mole, $3.78 \mathrm{~g})$ in DMF was taken in R.B.F. To it thiolactic acid $(0.01$ mole, $1.06 \mathrm{~g})$ was added slowly. Then it was placed inside a microwave oven for about 3.0 minutes. It was then diluted with ice cold water. The solid product formed was filtered, dried and recrystallised from Ethanol. Other substituted Thiazolidinones were prepared in a similar manner. Data for different substituted Thiazolidinones are given in Table-2.

Table 1 PHYSICAL \& ANALYTICAL DATA OF THE COMPOUNDS [A]

\begin{tabular}{|c|c|c|c|c|c|c|c|}
\hline \multirow[t]{2}{*}{ No. } & \multirow[t]{2}{*}{$\mathrm{R}$} & \multirow{2}{*}{$\begin{array}{c}\text { M.F. } \\
\text { (M.W.) }\end{array}$} & \multirow{2}{*}{$\begin{array}{l}\text { YIELD } \\
(\%)\end{array}$} & \multirow{2}{*}{$\begin{array}{l}\text { M.P. } \\
\left({ }^{\circ} \mathrm{C}\right)\end{array}$} & \multicolumn{3}{|c|}{$\begin{array}{c}\% \text { ANALYSIS } \\
\text { CALC.(FOUND) }\end{array}$} \\
\hline & & & & & $C$ & $\mathrm{H}$ & $\mathrm{N}$ \\
\hline BN-1 & $\begin{array}{c}3-\mathrm{OCH}_{3}, 4- \\
\mathrm{OH}\end{array}$ & $\begin{array}{c}\mathrm{C}_{22} \mathrm{H}_{16} \mathrm{~N}_{2} \mathrm{O}_{5} \mathrm{~S}_{2} \\
(452.0)\end{array}$ & 90 & 118 & $\begin{array}{c}58.40 \\
(58.42)\end{array}$ & $\begin{array}{c}3.53 \\
(3.51)\end{array}$ & $\begin{array}{c}6.19 \\
(6.23)\end{array}$ \\
\hline BN-2 & $2-\mathrm{NO}_{2}$ & $\begin{array}{c}\mathrm{C}_{21} \mathrm{H}_{13} \mathrm{~N}_{3} \mathrm{O}_{5} \mathrm{~S}_{2} \\
(451.0)\end{array}$ & 84 & 168 & $\begin{array}{l}55.87 \\
(55.90)\end{array}$ & $\begin{array}{l}2.88 \\
(2.86)\end{array}$ & $\begin{array}{c}9.31 \\
(9.34)\end{array}$ \\
\hline BN-3 & $2-\mathrm{a}$ & $\begin{array}{c}\mathrm{C}_{21} \mathrm{H}_{13} \mathrm{~N}_{2} \mathrm{O}_{3} \mathrm{~S}_{2} \mathrm{Cl} \\
(440.5)\end{array}$ & 82 & 126 & $\begin{array}{c}57.20 \\
(57.18)\end{array}$ & $\begin{array}{l}2.95 \\
(2.93)\end{array}$ & $\begin{array}{l}6.35 \\
(6.33)\end{array}$ \\
\hline BN-4 & $4-\mathrm{a}$ & $\begin{array}{c}\mathrm{C}_{21} \mathrm{H}_{13} \mathrm{~N}_{2} \mathrm{O}_{3} \mathrm{~S}_{2} \mathrm{Cl} \\
(440.5)\end{array}$ & 80 & 133 & $\begin{array}{l}57.20 \\
(57.24)\end{array}$ & $\begin{array}{l}2.95 \\
(2.97)\end{array}$ & $\begin{array}{c}6.35 \\
(6.39)\end{array}$ \\
\hline BN-5 & $2,4-(\mathrm{d})_{2}$ & $\begin{array}{c}\mathrm{C}_{21} \mathrm{H}_{12} \mathrm{~N}_{2} \mathrm{O}_{3} \mathrm{~S}_{2} \mathrm{C}_{2} \\
(475.0)\end{array}$ & 81 & 135 & $\begin{array}{l}53.05 \\
(53.03)\end{array}$ & $\begin{array}{l}2.52 \\
(2.54)\end{array}$ & $\begin{array}{c}5.89 \\
(5.94)\end{array}$ \\
\hline BN-6 & $2-\mathrm{OCH}_{3}$ & $\begin{array}{c}\mathrm{C}_{22} \mathrm{H}_{16} \mathrm{~N}_{2} \mathrm{O}_{4} \mathrm{~S}_{2} \\
(436.0)\end{array}$ & 86 & 165 & $\begin{array}{c}60.55 \\
(60.57)\end{array}$ & $\begin{array}{c}3.66 \\
(3.68)\end{array}$ & $\begin{array}{c}6.42 \\
(6.45)\end{array}$ \\
\hline BN-7 & $4-\mathrm{OCH}_{3}$ & $\begin{array}{c}\mathrm{C}_{22} \mathrm{H}_{16} \mathrm{~N}_{2} \mathrm{O}_{4} \mathrm{~S}_{2} \\
(436.0)\end{array}$ & 85 & 156 & $\begin{array}{c}60.55 \\
(60.58)\end{array}$ & $\begin{array}{c}3.66 \\
(3.63)\end{array}$ & $\begin{array}{c}6.42 \\
(6.40)\end{array}$ \\
\hline BN-8 & $\begin{array}{l}3,4,5- \\
\left(\mathrm{OCH}_{3}\right)_{3}\end{array}$ & $\begin{array}{c}\mathrm{C}_{24} \mathrm{H}_{20} \mathrm{~N}_{2} \mathrm{O}_{6} \mathrm{~S}_{2} \\
(496.0)\end{array}$ & 90 & 97 & $\begin{array}{l}58.06 \\
(58.10)\end{array}$ & $\begin{array}{l}4.03 \\
(4.06)\end{array}$ & $\begin{array}{c}5.64 \\
(5.62)\end{array}$ \\
\hline $\mathrm{BN}-9$ & $4-\mathrm{N}\left(\mathrm{CH}_{3}\right)_{2}$ & $\begin{array}{c}\mathrm{C}_{23} \mathrm{H}_{19} \mathrm{~N}_{3} \mathrm{O}_{3} \mathrm{~S}_{2} \\
(449.0)\end{array}$ & 84 & 151 & $\begin{array}{l}61.46 \\
(61.49)\end{array}$ & $\begin{array}{l}4.23 \\
(4.26)\end{array}$ & $\begin{array}{l}9.35 \\
(9.39)\end{array}$ \\
\hline BN-10 & $2-\mathrm{OH}$ & $\begin{array}{c}\mathrm{C}_{21} \mathrm{H}_{14} \mathrm{~N}_{2} \mathrm{O}_{4} \mathrm{~S}_{2} \\
(422.0)\end{array}$ & 87 & 144 & $\begin{array}{l}59.71 \\
(59.68)\end{array}$ & $\begin{array}{l}3.31 \\
(3.35)\end{array}$ & $\begin{array}{l}6.63 \\
(6.60)\end{array}$ \\
\hline
\end{tabular}


Table 2 PHYSICAL \& ANALYTICAL DATA OF THE COMPOUNDS [B]

\begin{tabular}{|c|c|c|c|c|c|c|c|}
\hline \multirow[t]{2}{*}{ NO. } & \multirow[t]{2}{*}{$\mathrm{R}$} & \multirow{2}{*}{$\begin{array}{l}\text { M.F. } \\
\text { (M.W.) }\end{array}$} & \multirow{2}{*}{$\begin{array}{l}\text { YIELD } \\
(\%)\end{array}$} & \multirow{2}{*}{$\begin{array}{l}\text { M.P. } \\
\left({ }^{\circ} \mathrm{C}\right)\end{array}$} & \multicolumn{3}{|c|}{$\begin{array}{l}\text { \% ANALYSIS } \\
\text { CALC.(FOUND) }\end{array}$} \\
\hline & & & & & $C$ & $\mathrm{H}$ & $\mathrm{N}$ \\
\hline BN-11 & $3-\mathrm{OCH}_{3}, 4-\mathrm{OH}$ & $\begin{array}{c}\mathrm{C}_{23} \mathrm{H}_{18} \mathrm{~N}_{2} \mathrm{O}_{5} \mathrm{~S}_{2} \\
(466.0)\end{array}$ & 85 & 161 & $\begin{array}{l}59.22 \\
(59.26)\end{array}$ & $\begin{array}{c}3.86 \\
(3.88)\end{array}$ & $\begin{array}{l}6.00 \\
(6.04)\end{array}$ \\
\hline BN-12 & $2-\mathrm{NO}_{2}$ & $\begin{array}{c}\mathrm{C}_{22} \mathrm{H}_{15} \mathrm{~N}_{3} \mathrm{O}_{5} \mathrm{~S}_{2} \\
(465.0)\end{array}$ & 80 & 186 & $\begin{array}{l}56.77 \\
(56.80)\end{array}$ & $\begin{array}{c}3.22 \\
(3.24)\end{array}$ & $\begin{array}{l}9.03 \\
(9.00)\end{array}$ \\
\hline BN-13 & $2-a$ & $\begin{array}{c}\mathrm{C}_{22} \mathrm{H}_{15} \mathrm{~N}_{2} \mathrm{O}_{3} \mathrm{~S}_{2} \mathrm{Cl} \\
(454.5)\end{array}$ & 75 & 158 & $\begin{array}{l}58.08 \\
(58.06)\end{array}$ & $\begin{array}{l}3.30 \\
(3.31)\end{array}$ & $\begin{array}{l}6.16 \\
(6.20)\end{array}$ \\
\hline BN-14 & $4-a$ & $\begin{array}{c}\mathrm{C}_{22} \mathrm{H}_{15} \mathrm{~N}_{2} \mathrm{O}_{3} \mathrm{~S}_{2} \mathrm{Cl} \\
(454.5)\end{array}$ & 74 & 164 & $\begin{array}{l}58.08 \\
(58.11)\end{array}$ & $\begin{array}{l}3.30 \\
(3.28)\end{array}$ & $\begin{array}{l}6.16 \\
(6.18)\end{array}$ \\
\hline BN-15 & $2,4-(\mathrm{d})_{2}$ & $\begin{array}{c}\mathrm{C}_{22} \mathrm{H}_{14} \mathrm{~N}_{2} \mathrm{O}_{3} \mathrm{~S}_{2} \mathrm{O}_{2} \\
(489.0)\end{array}$ & 78 & 167 & $\begin{array}{l}53.98 \\
(53.94)\end{array}$ & $\begin{array}{l}2.86 \\
(2.89)\end{array}$ & $\begin{array}{l}5.72 \\
(5.74)\end{array}$ \\
\hline BN-16 & $2-\mathrm{OCH}_{3}$ & $\begin{array}{c}\mathrm{C}_{23} \mathrm{H}_{18} \mathrm{~N}_{2} \mathrm{O}_{4} \mathrm{~S}_{2} \\
(450.0)\end{array}$ & 73 & 127 & $\begin{array}{l}61.33 \\
(61.29)\end{array}$ & $\begin{array}{c}4.00 \\
(3.98)\end{array}$ & $\begin{array}{l}6.22 \\
(6.24)\end{array}$ \\
\hline BN-17 & $4-\mathrm{OCH}_{3}$ & $\begin{array}{c}\mathrm{C}_{23} \mathrm{H}_{18} \mathrm{~N}_{2} \mathrm{O}_{4} \mathrm{~S}_{2} \\
(450.0)\end{array}$ & 77 & 117 & $\begin{array}{l}61.33 \\
(61.36)\end{array}$ & $\begin{array}{l}4.00 \\
(4.04)\end{array}$ & $\begin{array}{c}6.22 \\
(6.20)\end{array}$ \\
\hline BN-18 & $3,4,5-\left(\mathrm{OCH}_{3}\right)_{3}$ & $\begin{array}{c}\mathrm{C}_{25} \mathrm{H}_{22} \mathrm{~N}_{2} \mathrm{O}_{6} \mathrm{~S}_{2} \\
(510.0)\end{array}$ & 85 & 153 & $\begin{array}{l}58.82 \\
(58.84)\end{array}$ & $\begin{array}{c}4.31 \\
(4.29)\end{array}$ & $\begin{array}{l}5.49 \\
(5.53)\end{array}$ \\
\hline BN-19 & $4-\mathrm{N}\left(\mathrm{CH}_{3}\right)_{2}$ & $\begin{array}{c}\mathrm{C}_{24} \mathrm{H}_{21} \mathrm{~N}_{3} \mathrm{O}_{3} \mathrm{~S}_{2} \\
(463.0)\end{array}$ & 86 & 170 & $\begin{array}{l}62.20 \\
(62.22)\end{array}$ & $\begin{array}{c}4.53 \\
(4.56)\end{array}$ & $\begin{array}{l}9.07 \\
(9.09)\end{array}$ \\
\hline BN-20 & $2-\mathrm{OH}$ & $\begin{array}{c}\mathrm{C}_{22} \mathrm{H}_{16} \mathrm{~N}_{2} \mathrm{O}_{4} \mathrm{~S}_{2} \\
(436.0)\end{array}$ & 88 & 148 & $\begin{array}{l}60.55 \\
(60.57)\end{array}$ & $\begin{array}{c}3.66 \\
(3.69)\end{array}$ & $\begin{array}{r}6.42 \\
(6.43) \\
\end{array}$ \\
\hline
\end{tabular}

\section{Results and Discussion}

Structures of compounds synthesized have been elucidated by elemental analysis \& IR measurements. 4-Thiazolidinone compound shows IR absorption bands at $1330-1310 \mathrm{~cm}^{-1}\left(\mathrm{Ar}-\mathrm{CH}_{3}\right), 800-600 \mathrm{~cm}^{-1}$ (C-S stretching), $1720-1700 \mathrm{~cm}^{-1}$ (C=0 stretching) and $1360-1310 \mathrm{~cm}^{-1}$ (C-N stretching), $1690-1640 \mathrm{~cm}^{-1}$ $(\mathrm{C}=\mathrm{N})$.

ANTIBACTERIAL ACTIVITY:

This part deals with the in-vitro screening of newly synthesized compounds for their antimicrobial activity for filter paper disc method at a concentration of $50 \mathrm{ìg}$; the species, Staphylococcus aureus and Escherichia coli have been taken for the antibacterial activity. The compounds possess moderate to good activity against all stains in comparison with ampicillin, penicillin and tetracycline against Escherichia coli and Staphylococcus aureus. (Table-3)

TABLE - 3 ANTIBACTERIAL ACTIVITY OF NEWLY SYNTHESISED COMPOUNDS

\begin{tabular}{|c|c|c|c|c|c|}
\hline \multirow{2}{*}{ No. } & \multicolumn{2}{|c|}{ ZONE OF INHIBITION (mm) } & \multirow{2}{*}{ No. } & \multicolumn{2}{|c|}{ ZONE OF INHIBITION (mm) } \\
\hline & S.aureus. & E.coli. & & S.aureus. & E.coli. \\
\hline BN-1 & 11.0 & 9.0 & BN-11 & 13.0 & 11.0 \\
\hline BN-2 & 9.0 & 8.0 & $\mathrm{BN}-12$ & 9.0 & 8.0 \\
\hline $\mathrm{BN}-3$ & 9.0 & 7.0 & BN-13 & 6.0 & 8.0 \\
\hline
\end{tabular}


Contd..

\begin{tabular}{cllllll}
\hline \multirow{2}{*}{ No. } & \multicolumn{2}{c}{ ZONE OF INHIBITION $(\mathrm{mm})$} & \multirow{2}{*}{ No. } & \multicolumn{2}{c}{ ZONE OF INHIBITION (mm) } \\
\cline { 2 - 3 } & S.aureuS. & E.coli. & & S.aureus. & E.coli. \\
\hline BN-4 & 7.0 & 8.0 & BN-14 & 8.0 & 9.0 \\
BN-5 & 10.0 & 11.0 & BN-15 & 12.0 & 9.0 \\
BN-6 & 9.0 & 10.0 & BN-16 & 7.0 & 6.0 \\
BN-7 & 11.0 & 10.0 & BN-17 & 9.0 & 11.0 \\
BN-8 & 13.0 & 11.0 & BN-18 & 8.0 & 10.0 \\
BN-9 & 12.0 & 10.0 & BN-19 & 9.0 & 7.0 \\
BN-10 & 8.0 & 6.0 & BN-20 & 10.0 & 9.0 \\
\hline
\end{tabular}

\section{ACKNOWLEDGEMENT:}

We are grateful to the Head, Department of Chemistry, South Gujarat University, Surat for providing the necessary facilities for the research work. One of the author (BDNAIK) is also thankful to Dr.Hiren Vashi, Atul Limited, for providing chemicals for research work

\section{Reference}

1 Carpe E and Toma A, Chem Abstr., 1965, 63, 1484e.

2 Hans Swiss N, 1977, 592103; Chem. Abstr., 1978, 88, 22886n.

3 Warad D U, Satish CD, Kulkarni V H and Bajgur C S, Indian J Chem, 2000, 39A, 415.

4 Dash B, Mahapatra P K, Panda D and Patnaik J M, Indian Chem Soc, 1984, 61,1061.

5 Yadav R, Srivastava Soumya, Srivastava S K and Srivastava S D, Chemistry An Indian Journal, 2003, 1, 95

6 Desai P S, Desai K R, J Indian Chem Soc, 1994, 71, 155.

7 Fadayon M, Kulkarni V D and Pakdaman A SH, Asian J Chem, 1993, 5(2), 282.

8 Srivastava S K, Srivastava S and Srivastava SD, Indian J Chem, 1999, 38B, 183.

9 Bhatt J J, Shah B R, Trivedi P B, Yndavia N Kand Desai N C, Indian J Chem, 1994, 33B, 189.

10 Varma S, Green Chemistry, 1999, 1, 43.

11 Rostamizadeh S and Jafari S, Indian J Heterocyclic Chem, 2001, 10, 303.

12 Mogilaiah K, Reddy P R and Reddy N V, Indian J Heterocyclic Chem, 2001, 10, 267.

13 Cleophax J, Liagre M, Loupy A and Petit A, Org Process Res Dev, 2002, 6, 498. 


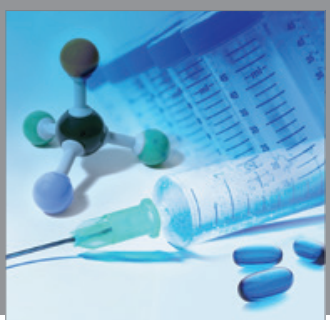

International Journal of

Medicinal Chemistry

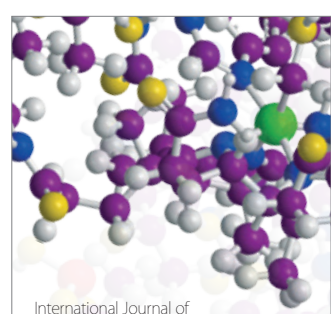

Carbohydrate Chemistry

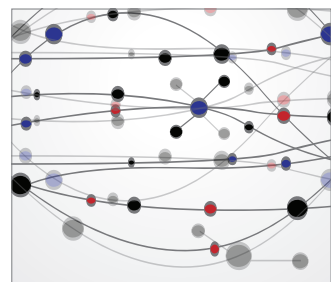

The Scientific World Journal
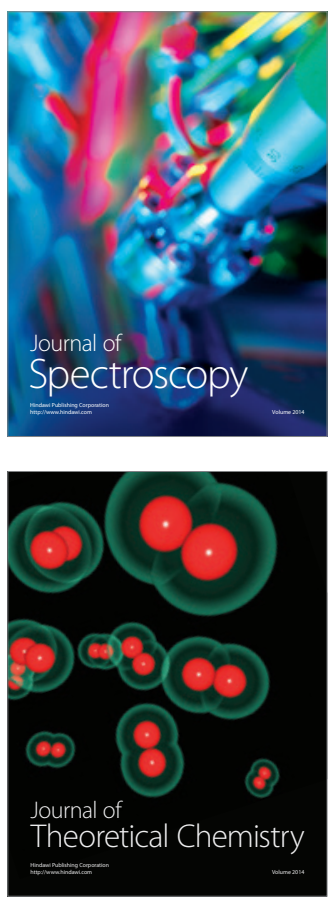
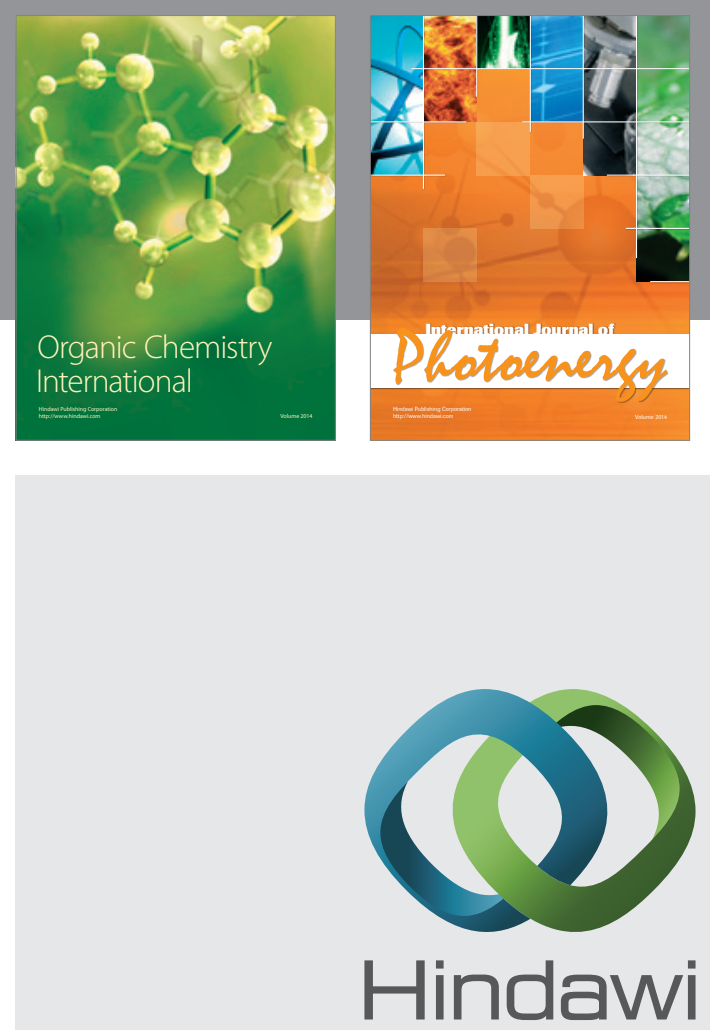

Submit your manuscripts at

http://www.hindawi.com
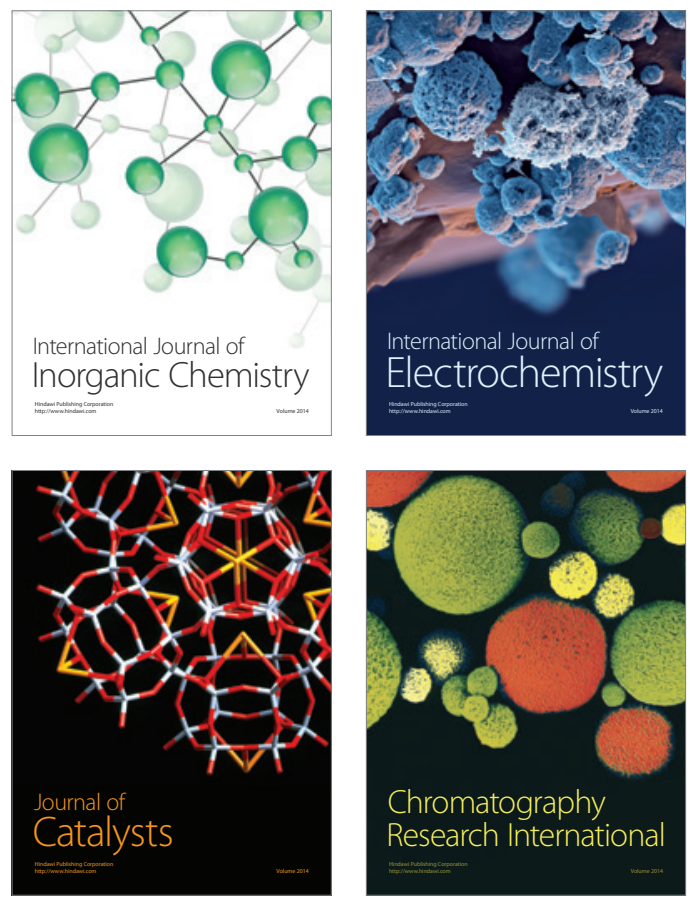
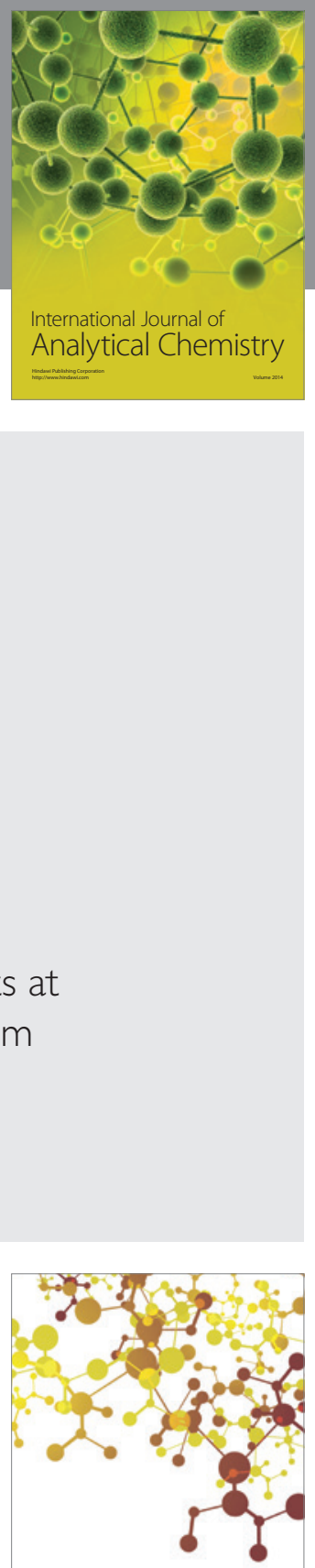

Journal of

Applied Chemistry
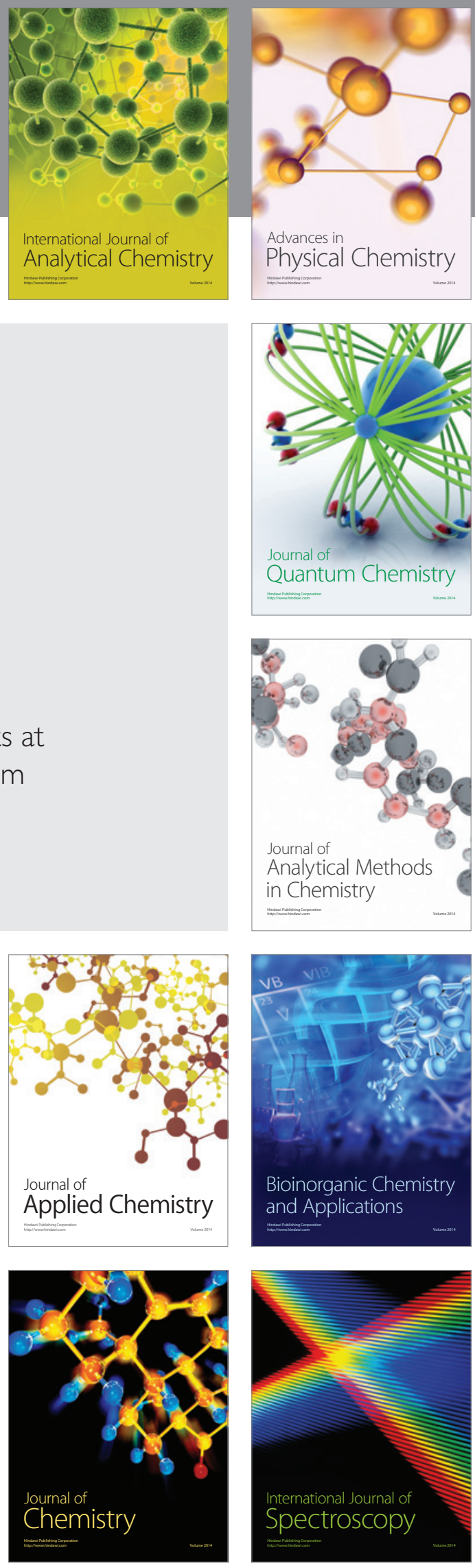